\title{
The volunteer's dilemma in finite populations
}

\author{
Kai A. Konrad ${ }^{1}$ (D) . Florian Morath ${ }^{2}$ (D)
}

Accepted: 26 December 2020 / Published online: 9 February 2021

(C) The Author(s) 2021

\begin{abstract}
We study the long-run stochastic stability properties of volunteering strategies in finite populations. We allow for mixed strategies, characterized by the probability that a player may not volunteer. A pairwise comparison of evolutionary strategies shows that the strategy with a lower probability of volunteering is advantaged. However, in the long run there are also populations of volunteering types. Monomorphisms with the more volunteering types are more frequent if the populations have fewer members, and if the benefits from volunteering are larger. Such monomorphisms with volunteering cease to exist if the population becomes infinitely large. In contrast, the disadvantage of volunteering disappears if the ratio of individual benefits and costs of volunteering becomes infinitely large.
\end{abstract}

Keywords Volunteering $\cdot$ Stochastic stability $\cdot$ Finite populations $\cdot$ Mixed strategies

JEL Classification C73 $\cdot$ D62 $\cdot$ H41

\section{Introduction}

Groups often suffer from the following problem known as the volunteer's dilemma: Several members of a group might have the option to take a costly action. If at least one of them volunteers and takes this action, this benefits all members in the group. The volunteer also benefits but-unlike the other members of the group-incurs a cost. So the volunteer's net advantage is lower than that of other group members. Each group member would be willing to volunteer if nobody else does, but each would prefer that someone else volunteers.

Kai A. Konrad

kai.konrad@tax.mpg.de

1 Max Planck Institute for Tax Law and Public Finance, Marstallplatz 1, 80539 Munich, Germany

2 University of Innsbruck, Universitätsstraße 15, 6020 Innsbruck, Austria 
Examples can be found in many contexts. Teams in military combat need volunteers for dangerous special tasks or for actions that might rescue the whole team. ${ }^{1}$ Firms choose whether to experiment, or to innovate technologies that have commongood nature, such that all firms of the same industry might learn or benefit from this volunteering activity. ${ }^{2}$ Single firms or their CEOs take lobbying actions that benefit these firms but might also be advantageous for the whole industry. ${ }^{3}$ Norm enforcement through social punishment is also a volunteering game: several players might observe a norm-violating behavior and think about whether to sanction the norm violator. The sanctioning activity is costly for the punisher and constitutes a contribution to the enforcement of collective rules, so each player has an incentive to abstain, hoping that someone else engages in this costly activity. ${ }^{4}$ The volunteer's dilemma also caught the attention of psychologists (see, e.g., Olivola et al. 2020). And the animal world provides further illustrative evidence. Archetti (2009a, b) alludes to a set of empirical environments in which the problem emerges. A generic example is the choice of whether to make alarm calls that are costly for the call maker but alert the larger group, for instance, of an approaching predator. ${ }^{5}$ Archetti and Scheurin (2011) study the volunteer's dilemma for the case in which the group benefit emerges only if multiple players volunteer. Mielke et al. (2019) used artificial dummies of snakes in a natural environment to study the possible motives of snake alarms. They considered multiple possible reasons for such behavior, including the motive of the alarm giving individual to signal his strength or fitness. They found results in line with the volunteer's dilemma game. A related variant of the volunteer's dilemma emerges in waiting games, i.e., when wait-and-see behavior becomes an option for the individuals in a group. ${ }^{6}$ A vivid example is the story of the murder of Catherine Susan Genovese. According to early newspaper reports, thirty-eight random bystanders allegedly watched or listened to it for thirty-five minutes before the first person called the police (see Gansberg 1964, in a New York Times article). ${ }^{7}$

Diekmann (1985) offers a Nash equilibrium analysis of the volunteer's problem if players maximize their expected material payoffs. In the symmetric equilibrium players typically mix between volunteering and not volunteering, making each other potential volunteer just indifferent about whether to volunteer or not. We study the stochastic dynamics of Diekmann's volunteer's dilemma in the long run in a finite population. At each point of time the population consists of potentially several types,

\footnotetext{
${ }^{1}$ Blomberg et al. (2009) describe how merit awards might be used to turn a prisoner's dilemma into a volunteer's dilemma in the context of heroic acts in military combat.

${ }^{2}$ See Johnson (2002) for a model of a volunteer game with incomplete information in the context of the development of open-source software.

${ }^{3}$ Barbieri et al. (2020) study volunteering in this context, allowing for a trade-off between free-riding on other members of the own group and preempting other groups on behalf of the own group.

${ }^{4}$ See Przepiorka and Diekmann (2018) for a discussion.

${ }^{5}$ Archetti (2011) reconsiders the alarm-call problem and studies the role of players' vigilance, i.e., the probability that a group member will observe that there is a predator approaching or not. Vigilance is a prerequisite of making an alarm call in this case.

${ }^{6}$ See Bliss and Nalebuff (1984), Fudenberg and Tirole (1986) and Barbieri et al. (2020) for game theory treatments of this and related volunteering games.

${ }^{7}$ Later studies report that the story is not supported by the available evidence and must be corrected on the basis of the facts (see, e.g., Manning et al. 2007).
} 
where an individual's type is described by the probability that this player volunteers. The type composition of the population is determined by a Markov process in which strong forces of selection and weak forces of mutation describe the transition probabilities between population states. We study the long-run properties of this process, using a framework developed by Fudenberg et al. (2006).

Finite population size is important for this concept, much like in Schaffer (1988), and may make spiteful strategies evolutionarily advantageous. ${ }^{8}$ But unlike Schaffer (1988), the concept developed by Fudenberg et al. (2006) looks at the long-run composition of finite populations. It allows for a pairwise comparison of two strategies with respect to their asymptotic success frequencies. ${ }^{9}$ We ask what the limit distribution of strategies is. As strategies that randomize between volunteering and not volunteering might yield superior outcomes (see, e.g., Bergstrom 2014), we allow for such mixing. For any two strategies we find that the limit distribution has two monomorphic populations of players. Groups might consist of players who all volunteer with the same positive probability - hence, there is some degree of cooperation in the limit distribution. Populations with the strategy type that volunteers less frequently turn out to be more likely than populations with the strategy type that volunteers more frequently, however. This evolutionary advantage of the less volunteering type holds for any pairwise comparison of volunteering strategies. The analysis of all pairs of evolutionary strategies drawn from a set that is a continuum of such strategies shows that there is a complete and transitive rank order on this set in the volunteer's dilemma game.

We also look at the comparative static properties. Volunteering groups are more likely if the material benefit from volunteering is larger. If the material benefit becomes very large in comparison to the cost of volunteering then, in a pairwise comparison of evolutionary strategies, the advantage of less cooperative types disappears so that monomorphic groups of players with higher or lower volunteering probability become equally likely. We also look at group size and find results suggesting that volunteering is more pronounced in smaller groups, i.e., if the positive spillovers from volunteering benefit a smaller group of other players. In the limit where the group size becomes infinitely large, only monomorphic groups of players who volunteer less frequently have a positive probability to exist in a pairwise comparison with any other strategy type.

\section{The model}

The state game Consider the following sequence of state games that follow the rules of Diekmann's (1985) volunteer's dilemma game. In a given period the population

\footnotetext{
${ }^{8}$ Schaffer's (1988) criterion of whether a newly emerging mutant does better than incumbents in an otherwise homogeneous finite population has been used in many contexts to explain status preferences (Eaton and Eswaran 2003), the emergence of property rights (Eswaran and Neary 2014), in-group favoritism and out-group spite (Eaton et al. 2011; Konrad and Morath 2012), aggression in conflict (Hehenkamp et al. 2010), and the inclination to start violent conflict (Konrad and Morath 2016).

${ }^{9}$ For applications of this framework see, e.g., Böttcher and Nagler (2016), Hauert and Imhof (2012), and Traulsen and Nowak (2006).
} 
consists of $m>1$ players. The state game has a population that consists of players who apply at most two different evolutionary strategies. Each player can either volunteer or not volunteer. We denote these two actions as $a \in\{v, n\}$. Like in many evolutionary games studied, these pure strategies relate to the players' types. Broadening the framework, we allow for player types that randomize between these two actions. Let $\sigma$ with $\sigma \in[0,1]$ denote the probability for not volunteering, i.e., for $a=n$. We also refer to players who randomize according to $\sigma$ as a player of type $\sigma$. The analysis considers all possible pairs $(\sigma, \hat{\sigma}) \in(0,1] \times[0,1)$ with $\sigma>\hat{\sigma} .^{10}$

The material payoffs are as follows. If at least one player volunteers then all players receive a benefit that is equal to $G>1$. Each player who volunteers has a cost of volunteering that is normalized to 1 , regardless of how many other players volunteer. The name of the game is motivated by the observation that each player can increase her own material payoff by volunteering if no other player volunteers. But each player prefers not to volunteer if at least one of the other players volunteers. Let $s$ players choose the mixed strategy $\sigma$, i.e., they do not volunteer with a probability $\sigma$. Furthermore, let the other $(m-s)$ players follow mixed strategy $\hat{\sigma}$ such that each of them does not volunteer with probability $\hat{\sigma}$, where $\hat{\sigma}<\sigma$. Then, the player who follows the mixed strategy $\sigma$ has the expected material payoff

$$
y(s)=-(1-\sigma)+\left(1-\sigma^{s} \hat{\sigma}^{(m-s)}\right) G .
$$

Here, $(1-\sigma)$ is the expected cost from volunteering for a player of type $\sigma$. The term $\left(1-\sigma^{s} \hat{\sigma}^{(m-s)}\right)$ describes the probability that the public benefit $G$ will emerge if there are $s$ players of type $\sigma$ and $(m-s)$ players of type $\hat{\sigma}$, assuming that their actions emerge from their mixed strategies and in a way that makes their choice outcomes from these mixed strategies stochastically independent. Analogously, each player who follows strategy $\hat{\sigma}$ has the expected material payoff

$$
\hat{y}(s)=-(1-\hat{\sigma})+\left(1-\sigma^{s} \hat{\sigma}^{(m-s)}\right) G .
$$

These material payoffs describe player types' fitness in the state game. Since $G>1$, the expected material payoffs in Eqs. 1 and 2 are strictly positive.

Markov process We next turn to describing the population dynamics in an infinite sequence of periods $t=1,2,3, \ldots$, where a state game takes place in each of these periods. Let $h_{t}=s$ players behave according to $\sigma$ and $(m-s)$ players behave according to $\hat{\sigma}$ in period $t$. Recall that $\sigma>\hat{\sigma}$ by suitably naming the two strategies. The material payoff of a player in period $t$ does not depend on the actual period but only on the type composition of the population and the player's own type in the state game taking place in that period. For two given strategies $\sigma$ and $\hat{\sigma}$ in period $t$ all relevant parameters for how the dynamics evolve are fully described by these strategies and the single parameter $h_{t}$, that is, by the number of players who follow strategy $\sigma$. The types' expected material payoffs as in Eqs. 1 and 2 drive this Markov

\footnotetext{
${ }^{10} \mathrm{~A}$ motivating question for this generalization is whether the existence of mixed strategy types might cause stochastically stable bimorphisms. We limit consideration to pairwise comparisons. Extending the analysis to an infinite number of types would require different tools to deal with the stochastic dynamics with a continuum of types than we use here.
} 
process which follows the rules described by Fudenberg et al. (2006): The process is initially in the state $h_{0} \in\{0,1,2, \ldots, m\}$. From one period to the next the total number of individuals in the group remains fixed and equal to $m$ but one individual is chosen to reproduce. The transition probabilities $p_{s, s^{\prime}}$ between states $s$ and $s^{\prime}$ from period $t$ to period $t+1$ for a given state $h_{t}=s$ are described by Eqs. 3 to 8 . For interior states $s \in\{1, \ldots, m-1\}$ the Markov state remains unchanged with probability $p_{s, s}$ which equals

$$
p_{s, s}=1-p_{s, s-1}-p_{s, s+1},
$$

where $p_{s, s+1}$ and $p_{s, s-1}$ are the probabilities of transition to the neighboring states. Other transitions are not feasible:

$$
p_{s, s^{\prime}}=0 \text { for all } s^{\prime} \notin\{s-1, s, s+1\} .
$$

For the monomorphic boundary states $s=0$ and $s=m$ the process either stays there, or moves to the neighboring state in the interior. This is possible due to mutations, where $\mu$ measures the mutation probability.

$$
\begin{gathered}
p_{m, m}=1-p_{m, m-1}=1-\mu, \\
p_{0,0}=1-p_{0,1}=1-\mu,
\end{gathered}
$$

The probability for transitions from an interior state to one of the two neighboring states is given by

$$
p_{s, s+1}=\frac{s y(s)(1-\mu)+(m-s) \hat{y}(s) \mu}{s y(s)+(m-s) \hat{y}(s)} \frac{m-s}{m},
$$

and

$$
p_{s, s-1}=\frac{s y(s) \mu+(m-s) \hat{y}(s)(1-\mu)}{s y(s)+(m-s) \hat{y}(s)} \frac{s}{m} .
$$

The transition probabilities strictly follow Fudenberg et al. (2006). They depend on the material payoffs $y(s)$ and $\hat{y}(s)$, the frequency distribution of types and the probability $\mu \geq 0$ that a mutation will occur (i.e., that, for instance, an individual of type $\sigma$ mutates and becomes a type who volunteers according to $\hat{\sigma}$ instead of $\sigma$ ). Note that (3)-(8) describe the dynamics as a stochastic process, not as a deterministic process, as is commonly done in the context of populations with more than countably many individuals.

The probabilities in Eqs. 7 and 8 have an interpretation that becomes more transparent for the case $\mu=0$. It goes back to Moran's (1962) population dynamics with frequency dependent transition probabilities, as described in Nowak et al. (2004) and Taylor et al. (2004): all but one of the $m$ players simply survive into the next period (or reproduce identically) and keep their types. One of the $m$ players is singled out, 'dies' and is replaced by a player who is possibly of the same type or of the other type. To change the state from $h_{t}=s$ to $h_{t+1}=s+1$, the player who is singled out must necessarily be of type $\hat{\sigma}$ and must be replaced by a player of type $\sigma$. In the absence of mutations $(\mu=0)$ the probability of this happening reduces to the 
textbook version of frequency-dependent transition dynamics in the Moran process without mutations (see Nowak 2006, p. 109):

$$
\frac{s y(s)}{s y(s)+(m-s) \hat{y}(s)} \frac{m-s}{m} .
$$

Intuitively, any of the $m$ players in period $t$ can be the one who dies. The term $\frac{m-s}{m}$ is the probability that the player who dies is of type $\hat{\sigma}$. Furthermore, $s y /(s y+(m-s) \hat{y})$ describes the probability that the replacement is a player of type $\sigma$. This probability is frequency and fitness dependent. For $y=\hat{y}$ this is as likely as $s / m$, i.e., proportional to the share of type $\sigma$-players in the total population. But for $y \neq \hat{y}$ the selection of the replacement type follows a drift. For $y>\hat{y}$ this drift favors replacement by a player of type $\sigma$, and for $y<\hat{y}$ it favors replacement by a player of type $\hat{\sigma}$. The possibility of mutations modifies this transition probability from Eqs. 9 to 7 .

The reasoning for Eq. 8, i.e., the transition from $h_{t}=s$ to a state with $h_{t+1}=s-1$, i.e., with one player of type $\sigma$ being replaced by a player of type $\hat{\sigma}$, is analogous. If the process is in $h_{t}=s$ and does not transit to one of the neighboring states, it must stay at $s$, i.e., $h_{t+1}=s$, and the probability of this is, hence, (3). Without mutations $(\mu=0)$ the process (3)-(8) has two absorbing states, characterized by $s=0$ and $s=m$ and transition probabilities $p_{0,0}=p_{m, m}=1$. But mutation allows the process to leave these absorbing states, where we can think of $\mu$ as the probability of such an event. Also, in the non-absorbing states $s \in\{1,2, \ldots,(m-1)\}$ such a mutation (from $\sigma$ to $\hat{\sigma}$ or vice versa) might happen, as described in Eqs. 7 and 8.

The limit result The Markov process described by Eqs. 3 to 8 is an ergodic Markov chain: it is aperiodic and positive recurrent. Let $\mathbf{P}$ be the matrix of transition probabilities $p_{s, s^{\prime}}$. Because the Markov chain is ergodic, there exists a unique probability vector $\pi=\left(\pi_{1}, \ldots, \pi_{m}\right)$ that solves the equation

$$
\pi(\mu) \mathbf{P}=\pi(\mu)
$$

for any given positive $\mu$. The vector $\pi$ characterizes the unique limit distribution of this Markov chain, with the following interpretation: If analogous Markov processes operate in many populations in parallel-all of them following the logic of transition probabilities (3)-(8) and all running for a very long time-and if we look at these processes after a long enough time and identify the states in which these processes are in the different populations, then the limit probability $\pi_{S}(\sigma, \hat{\sigma})$ is the share of these populations that are in state $s$. The analytic steps in Fudenberg et al. (2006, p. 354) can be used to find the limit properties:

$$
\pi_{m}^{*} \equiv \lim _{\mu \rightarrow 0} \pi_{m}(\sigma, \hat{\sigma})=\frac{\gamma}{\gamma+1} \text { and } \pi_{0}^{*}=1-\pi_{m}^{*},
$$

where

$$
\gamma=\prod_{s=1}^{m-1} \frac{y(s)}{\hat{y}(s)} .
$$

We can, hence, state the following result: 
Proposition 1 Let the set of possible strategies be $\{\sigma, \hat{\sigma}\}$. (i) In the limit distribution for $\mu \rightarrow 0$ the population consists of players exclusively of type $\sigma$ with probability

$$
\pi_{m}^{*}(\sigma, \hat{\sigma})=\frac{\prod_{s=1}^{m-1} \frac{y(s)}{\hat{y}(s)}}{\prod_{s=1}^{m-1} \frac{y(s)}{\hat{y}(s)}+1}
$$

and of players exclusively of type $\hat{\sigma}$ with probability $1-\pi_{m}^{*}(\sigma, \hat{\sigma})$. (ii) $\pi_{m}^{*}(\sigma, \hat{\sigma})>$ $1 / 2$ if and only if $\sigma>\hat{\sigma}$. (iii) Let three feasible strategies be $\sigma_{1}, \sigma_{2}$, and $\sigma_{3}$. It holds that

$$
\left\{\pi_{m}^{*}\left(\sigma_{1}, \sigma_{2}\right)>\frac{1}{2} \text { and } \pi_{m}^{*}\left(\sigma_{2}, \sigma_{3}\right)>\frac{1}{2}\right\} \Longrightarrow \pi_{m}^{*}\left(\sigma_{1}, \sigma_{3}\right)>\frac{1}{2} .
$$

In words, if strategy $\sigma_{1}$ is advantageous against strategy $\sigma_{2}$ and $\sigma_{2}$ is advantageous against strategy $\sigma_{3}$, then $\sigma_{1}$ is advantageous against $\sigma_{3}$.

A proof of this and all further propositions is in the Appendix. The first part of the proposition suggests that, in the long run, the Markov process is either in state $s=0$ or in $s=m$ with probability 1 . Hence, if one wants to compare strategies with respect to their stochastic stability advantage, it makes sense to compare $\pi_{m}$ and $\pi_{0}$. Strategy $\sigma$ can be considered to be more advantageous than strategy $\hat{\sigma}$ if $\pi_{m}(\sigma, \hat{\sigma})>1 / 2$.

The characteristic of a strategy to be more advantageous mainly depends on material payoff relative to the material payoff associated with other strategies on the whole set of states. Consider a player who volunteers. In some situations the player is not pivotal, because one or several other players volunteer. Her action as a volunteer is redundant and only costly to her, making her worse off than others. In other situations the player is pivotal and $G$ is provided if and only if the player volunteers. If the player volunteers this increases the player's own material payoff by $G-1$. This increase is, however, smaller than the increase $G$ that results for all other members of the population. Volunteering does never improve the player's payoff relative to the payoffs of others and this provides an intuition for result (iii) in the proposition. It follows from (iii) that $\sigma=1$ (i.e., not volunteering with probability one) is the most advantageous evolutionary strategy, independent of which of the infinite number of other strategies might be the alternative strategy. This property is also interesting from a methodological point. It shows that there is a complete and transitive ordering in a pairwise comparison of strategies in the volunteer's dilemma. ${ }^{11}$

Stochastic stability versus evolutionary stability: an example Think of populations with two individuals only $(m=2)$. Consider a numerical example with a value of the public good of $G=2$. There is only one interior state, which is $s=1$. Inserting

\footnotetext{
${ }^{11}$ The fact that a lower volunteering probability (i.e., higher $\sigma$ ) is more advantageous could also be described as spiteful behavior: the player refrains from an action that would increase the material payoff of the player, and this behavior is chosen because the action would increase the material payoff of other players by even more.
} 
into (13) yields

$$
\pi_{m}^{*}=\frac{1-2 \sigma \hat{\sigma}+\sigma}{2-4 \sigma \hat{\sigma}+\sigma+\hat{\sigma}}
$$

as the probability that the population consists of players of type $\sigma$ only. We plot this probability for any combination $(\sigma, \hat{\sigma}) \in(0,1) \times(0,1)$ in Fig. 1 . It shows that for any $\sigma$ this probability is increasing in $\sigma$ and decreasing in $\hat{\sigma}$ with lower bound $\pi_{m}^{*}=\frac{1}{3}$ and upper bound $\pi_{m}^{*}=\frac{2}{3}$, and $\pi_{m}^{*}(\sigma, \hat{\sigma})=\frac{1}{2}$ for all $\sigma=\hat{\sigma}$.

Fudenberg et al. (2006) provides us with a framework to consider stochastic stability if the set of possible player types has only two elements, as in this example. In a more general framework a dynamic process might start in a monomorphic state characterized by $\sigma$. After a mutant $\hat{\sigma}$ emerges it might return to the monomorphic state $\sigma$, or it might transit into a monomorphic state characterized by $\hat{\sigma}$. It stays there until another mutant type $\tilde{\sigma} \in[0,1]$ emerges, which might differ from $\sigma$ and $\hat{\sigma}$, and so on. It would be interesting to characterize a possible surface of the limit distribution for any combination $(\sigma, \sigma)$ on the type space $[0,1] \times[0,1]$ if it exists, but we have to leave this problem to future analysis. Figure 1 is suggestive, showing a stochastic stability advantage of more selfishness, for whatever are the two types that might interact with each other.

To emphasize the divergence of this result to evolutionary stability in large populations, suppose that only the pure strategies $\sigma=0$ and $\sigma=1$ are feasible strategies. By Proposition 1 it then holds almost always that both members of the population either both volunteer or both do not volunteer. The state in which both individuals are of the non-volunteering type is more likely than the state in which both volunteer. For $m=2$, the probability that the population consists of non-volunteers only is $G /(2 G-1)$. We might compare the stochastic stability outcome in this case with the evolutionarily stable outcome if players in each period are drawn from a large set with more than countably many players that are teamed up randomly in pairwise interactions. If, in the latter environment, evolutionary strategies are restricted to the two pure strategies $\sigma=0$ and $\sigma=1$, an equilibrium in evolutionarily stable strategies has a share of $q=1 / G$ of players of type $\sigma=1$ and a remaining share of $(1-q)$

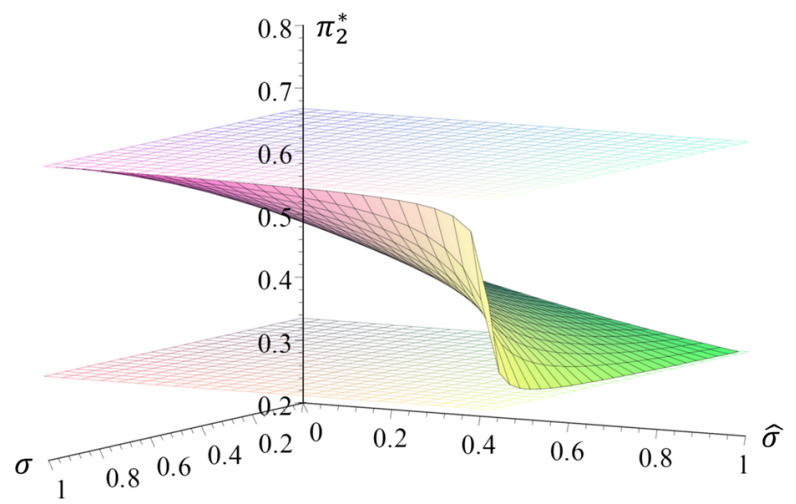

Fig. 1 Limit probability $\pi_{2}^{*}$ (on the vertical axis) for combinations of $\sigma$ and $\hat{\sigma}$ (on the horizontal axes). The horizontal planes at altitude $\frac{1}{3}$ and $\frac{2}{3}$ suggest that all $\pi_{2}^{*}$ are between these values 
of players of type $\sigma=0$. By construction of $q$, a player of type $\sigma=0$ has, in expectation, the material payoff of $G-1$. A player of type $\sigma=1$ has an expected material payoff of $(1-q) G$. These expected values are equal if $q=1 / G$. Any population mixture $\tilde{q}>q$ with a higher share of non-volunteering types favors the volunteering type $\sigma=0$ as $(1-\tilde{q}) G<(1-q) G=G-1$, and $G-1$ is the payoff of type $\sigma=0$. Similarly, any population mixture $\tilde{q}<q$ with a larger share of volunteering types favors the non-volunteering type $\sigma=1$ as $G-1=(1-q) G<(1-\tilde{q}) G$.

The divergence between evolutionary stability for infinitely large populations and stochastic stability of the Moran process as limit probabilities of the stochastic dynamics in finite populations has been noticed for some time. Transition dynamics of the underlying Markov process in finite populations are stochastic, whereas the evolutionary dynamics in infinite populations are typically approximated by deterministic processes. Foster and Young (1990) discuss the importance of an 'adequate' account of stochastic effects and suggest the concept of stochastically stable equilibrium. Our results fundamentally build on the limit results for ergodic Markov chains and the tool provided by Fudenberg et al. (2006) for calculating the invariant distribution. ${ }^{12}$ Attempts have been made by Harper and Fryer (2016) and Mohseni (2019) to reconcile stochastic stability in finite but large populations and standard concepts of deterministic approximiations of the dynamics of evolutionary processes in infnitely large populations.

If the dilemma becomes more severe We can study how the stationary group composition is affected by the relationship between the cost of volunteering (that was normalized to 1) and the individual gross benefit for each group member denoted by $G$. Recall that a higher $\sigma$ means that the strategy type volunteers less frequently and state $m$ means that the population exclusively consists of types $\sigma$ with $\sigma>\hat{\sigma}$.

Proposition 2 Suppose only two evolutionary strategies exist, characterized by $\sigma$ and $\hat{\sigma}$ with $\sigma>\hat{\sigma}$. The limit probability $\pi_{m}^{*}(\sigma, \hat{\sigma} ; G)$ for the Markov process to be in state $s=m$ is smaller if the group members' benefits $G$ from the volunteer's action is larger. Furthermore, $\lim _{G \rightarrow \infty} \pi_{m}^{*}(\sigma, \hat{\sigma} ; G)=\lim _{G \rightarrow \infty} \pi_{0}^{*}(\sigma, \hat{\sigma} ; G)=1 / 2$.

Proposition 2 suggests that groups of players who volunteer more frequently are more common in the limit distribution of group types if the benefit $G$ that each group member has if one group member volunteers is larger. A higher $G$ reduces the relative disadvantage which the volunteer has, compared to the non-volunteer. Accordingly, the two evolutionary strategies $\sigma$ and $\hat{\sigma}$ perform more equally in terms of relative material payoff and differences in the likelihoods $\pi_{m}^{*}$ and $\pi_{0}^{*}$ of monomorphic groups of each of the two strategy types are reduced. If the benefit $G$ becomes very large, the comparative disadvantage of being a volunteer becomes less and less important

\footnotetext{
${ }^{12}$ Their theorem draws on a theorem that was developed by Fudenberg and Imhof (2006) who relate their work to Freidlin and Wentzell (1984) on systems with random dynamics but offer an independent proof for their result.
} 
and vanishes in the limit so that monomorphic groups of more frequent volunteers and groups of less frequent volunteers become equally likely for $G \rightarrow \infty$.

Group size effects To study group size effects we consider the limit probability $\pi_{m}^{*}(\sigma, \hat{\sigma})$ when comparing a mixed strategy to a strategy of always or never volunteering.

Proposition 3 Suppose that $1=\sigma>\hat{\sigma}$ or $\sigma>\hat{\sigma}=0$. Then $\pi_{m}^{*}(\sigma, \hat{\sigma})$ is higher for higher $m$. For any $\sigma>\hat{\sigma}$, in the limit for very large groups, groups consist of the less volunteering types with probability 1 .

We find that, compared to any mixed strategy, the advantage of never volunteering as well as the disadvantage of always volunteering increase with group size. ${ }^{13}$ In the limit for large groups, the stationary distribution attributes a probability of 1 to groups that consist of less volunteering members only. The intuition for the result is again that only relative material success matters. It is precisely the fact that volunteering benefits others that is to the disadvantage of the volunteer. Hence, if more players benefit from the act of volunteering, then this disadvantage becomes larger.

\section{Conclusions}

We studied the volunteer's dilemma when the space of evolutionary strategies includes the two pure strategies (volunteer; not volunteer) and any mixing between these. We provide a pairwise comparison of all possible mixed strategies with respect to their long-run evolutionary advantage. This pairwise comparison builds on methods introduced by Fudenberg et al. (2006) for games with only two strategies. The pairwise comparison between all possible evolutionary strategies in a continuous strategy space does not necessarily lead to a complete ordering of strategies. However, in the context of the volunteer's dilemma, our analysis shows that such a complete ordering exists. Comparing any two strategies, the type who volunteers with a lower probability has a greater evolutionary advantage for any pairwise comparison.

\section{Appendix}

Proof of Proposition 1 (i) The first part of this proposition just inserts the descriptions of players' fitness (1) and (2) that emerge for the volunteer's dilemma in the

\footnotetext{
${ }^{13}$ Intuitively, similar comparative statics properties should hold for arbitrary pairs $(\sigma, \hat{\sigma})$ but analytical results are less straightforward in this case. The reason is that, when adding another player, the relative advantage from a lower volunteering probability decreases (material payoff increases for both strategies $\sigma$ and $\hat{\sigma}$ because it becomes more likely that someone else volunteers). However, volunteering benefits a larger group of players, which makes non-volunteering more attractive.
} 
machinery of Fudenberg et al. (2006). (ii) For the second part, note that

$$
\prod_{s=1}^{m-1} \frac{y(s)}{\hat{y}(s)}=\prod_{s=1}^{m-1} \frac{z(s)+\sigma}{z(s)+\hat{\sigma}}
$$

where

$$
z(s)=\left(-1+\left(1-\sigma^{s} \hat{\sigma}^{m-s}\right) G\right) .
$$

We can re-write (13) as

$$
\pi_{m}^{*}=\frac{\prod_{s=1}^{m-1} \frac{z(s)+\sigma}{z(s)+\hat{\sigma}}}{\prod_{s=1}^{m-1} \frac{z(s)+\sigma}{z(s)+\hat{\sigma}}+1} .
$$

Hence, $\pi_{m}^{*}$ is larger than $1 / 2$ if and only if

$$
\prod_{s=1}^{m-1} \frac{z(s)+\sigma}{z(s)+\hat{\sigma}}>1 .
$$

This inequality holds because, for each single factor, $(z(s)+\sigma) /(z(s)+\hat{\sigma})>1$ if and only if $\sigma>\hat{\sigma}$. Thus, $\sigma>\hat{\sigma}$ is necessary and sufficient for $\pi_{m}^{*}(\sigma, \hat{\sigma})>1 / 2$. (iii) By part (ii), $\pi_{m}^{*}\left(\sigma_{1}, \sigma_{2}\right)>1 / 2$ requires $\sigma_{1}>\sigma_{2}$ and $\pi_{m}^{*}\left(\sigma_{2}, \sigma_{3}\right)>1 / 2$ requires $\sigma_{2}>\sigma_{3}$. Thus, $\sigma_{1}>\sigma_{3}$, and this, in turn, implies $\pi_{m}^{*}\left(\sigma_{1}, \sigma_{3}\right)>1 / 2$.

Proof of Proposition 2 Equation 13 can be transformed to

$$
\frac{1}{\pi_{m}^{*}(\sigma, \hat{\sigma} ; G)}=1+\frac{1}{\prod_{s=1}^{m-1} \frac{\left(\left(1-\sigma^{s} \hat{\sigma}^{m-s}\right) G-1\right)+\sigma}{\left(\left(1-\sigma^{s} \hat{\sigma}^{m-s}\right) G-1\right)+\hat{\sigma}}} .
$$

Hence, $\pi_{m}^{*}(\sigma, \hat{\sigma} ; G)$ is decreasing if

$$
\frac{\partial\left[\prod_{s=1}^{m-1} \frac{\left(\left(1-\sigma^{s} \hat{\sigma}^{m-s}\right) G-1\right)+\sigma}{\left(\left(1-\sigma^{s} \hat{\sigma}^{m-s}\right) G-1\right)+\hat{\sigma}}\right]}{\partial G}<0 .
$$

Inequality (21) holds because

$$
\frac{\partial\left[\frac{\left(\left(1-\sigma^{s} \hat{\sigma}^{m-s}\right) G-1\right)+\sigma}{\left(\left(1-\sigma^{s} \hat{\sigma}^{m-s}\right) G-1\right)+\hat{\sigma}}\right]}{\partial G}=-\frac{\left(1-\sigma^{s} \hat{\sigma}^{m-s}\right)(\sigma-\hat{\sigma})}{\left(G\left(1-\sigma^{s} \hat{\sigma}^{m-s}\right)+\hat{\sigma}-1\right)^{2}}<0
$$

for each factor in the product. The limit property for $G \rightarrow \infty$ then follows directly from

$$
\lim _{G \rightarrow \infty} \frac{1}{\pi_{m}^{*}(\sigma, \hat{\sigma} ; G)}=1+\lim _{G \rightarrow \infty} \frac{1}{\prod_{s=1}^{m-1} \frac{\left(\left(1-\sigma^{s} \hat{\sigma}^{m-s}\right) G-1\right)+\sigma}{\left(\left(1-\sigma^{s} \hat{\sigma}^{m-s}\right) G-1\right)+\hat{\sigma}}}=2 .
$$


Proof of Proposition 3 We have to show that, for $\tilde{m}=m+1$ the inequality $\frac{1}{\pi_{m}^{*}}>\frac{1}{\pi_{\tilde{m}}^{*}}$ holds if $\sigma=1>\hat{\sigma}$ or $\sigma>\hat{\sigma}=0$, where

$$
\frac{1}{\pi_{m}^{*}(\sigma, \hat{\sigma})}=1+\prod_{s=1}^{m-1} \frac{\left(\left(1-\sigma^{s} \hat{\sigma}^{m-s}\right) G-1\right)+\hat{\sigma}}{\left(\left(1-\sigma^{s} \hat{\sigma}^{m-s}\right) G-1\right)+\sigma}
$$

and

$$
\frac{1}{\pi_{\tilde{m}}^{*}(\sigma, \hat{\sigma})}=1+\prod_{s=1}^{m+1-1} \frac{\left(\left(1-\sigma^{s} \hat{\sigma}^{m+1-s}\right) G-1\right)+\hat{\sigma}}{\left(\left(1-\sigma^{s} \hat{\sigma}^{m+1-s}\right) G-1\right)+\sigma} .
$$

Consider first the case $\sigma=1$. The inequality $\frac{1}{\pi_{m}^{*}}>\frac{1}{\pi_{\tilde{m}}^{*}}$ reduces to

$$
\prod_{s=1}^{m-1} \frac{\left(\left(1-\hat{\sigma}^{m-s}\right) G-1\right)+\hat{\sigma}}{\left(\left(1-\hat{\sigma}^{m-s}\right) G-1\right)+1}>\prod_{s=1}^{m+1-1} \frac{\left(\left(1-\hat{\sigma}^{m+1-s}\right) G-1\right)+\hat{\sigma}}{\left(\left(1-\hat{\sigma}^{m+1-s}\right) G-1\right)+1}
$$

The right-hand side of this inequality can be written as

$$
\begin{aligned}
& \frac{\left(\left(1-\hat{\sigma}^{m+1-1}\right) G-1\right)+\hat{\sigma}}{\left(\left(1-\hat{\sigma}^{m+1-1}\right) G-1\right)+1} \prod_{s=2}^{m+1-1} \frac{\left(\left(1-\hat{\sigma}^{m+1-s}\right) G-1\right)+\hat{\sigma}}{\left(\left(1-\hat{\sigma}^{m+1-s}\right) G-1\right)+1} \\
& =\frac{\left(\left(1-\hat{\sigma}^{m+1-1}\right) G-1\right)+\hat{\sigma}}{\left(\left(1-\hat{\sigma}^{m+1-1}\right) G-1\right)+1} \prod_{s=1}^{m-1} \frac{\left(\left(1-\hat{\sigma}^{m-s}\right) G-1\right)+\hat{\sigma}}{\left(\left(1-\hat{\sigma}^{m-s}\right) G-1\right)+1}
\end{aligned}
$$

and the claim then follows from $\frac{\left(\left(1-\hat{\sigma}^{m+1-1}\right) G-1\right)+\hat{\sigma}}{\left(\left(1-\hat{\sigma}^{m+1-1}\right) G-1\right)+1}<1$.

Consider next the case $\hat{\sigma}=0$. The comparison simplifies to

$$
\prod_{s=1}^{m-1} \frac{G-1}{G-1+\sigma}>\prod_{s=1}^{m+1-1} \frac{G-1}{G-1+\sigma}
$$

and holds because

$$
\frac{G-1}{G-1+\sigma} \in(0,1)
$$

The limit property (ii) follows from

$$
\lim _{m \rightarrow \infty} \pi_{m}^{*}=\frac{1}{1+\lim _{m \rightarrow \infty} \prod_{s=1}^{m-1} \frac{\left(\left(1-\sigma^{s} \hat{\sigma}^{m-s}\right) G-1\right)+\hat{\sigma}}{\left(\left(1-\sigma^{s} \hat{\sigma}^{m-s}\right) G-1\right)+\sigma}}=1
$$

for $\sigma>\hat{\sigma}$.

Acknowledgments We thank two anonymous reviewers for very helpful comments and suggestions. The usual caveat applies.

Funding Open Access funding enabled and organized by Projekt DEAL. This study was funded as part of our academic employments. No specific funding for the project was received.

\section{Declarations}

Research involving human participants and/ or animals This study does not involve research involving human participants or animals. 
Ethical approval Does not apply. This work does not contain any studies with human participants or animals performed by any of the authors.

Informed consent Does not apply. There were no individual participants included in the study.

Conflict of interests The authors declare that they have no conflict of interest that they are aware of.

Open Access This article is licensed under a Creative Commons Attribution 4.0 International License, which permits use, sharing, adaptation, distribution and reproduction in any medium or format, as long as you give appropriate credit to the original author(s) and the source, provide a link to the Creative Commons licence, and indicate if changes were made. The images or other third party material in this article are included in the article's Creative Commons licence, unless indicated otherwise in a credit line to the material. If material is not included in the article's Creative Commons licence and your intended use is not permitted by statutory regulation or exceeds the permitted use, you will need to obtain permission directly from the copyright holder. To view a copy of this licence, visit http://creativecommonshorg/licenses/by/4. $0 /$.

\section{References}

Archetti M (2009a) The volunteer's dilemma and the optimal size of a social group. J Theor Biol 261(3):475-480

Archetti M (2009b) Cooperation as a volunteer's dilemma and the strategy of conflict in public goods games. J Evol Biol 22(11):2192-2200

Archetti M (2011) A strategy to increase cooperation in the volunteer's dilemma: reducing vigilance improves alarm calls. Evolution 65(3):885-892

Archetti M, Scheurin I (2011) Coexistence of cooperation and defection in public goods games. Evolution 65(4):1140-1148

Barbieri S, Konrad KA, Malueg DA (2020) Preemption contests between groups, RAND Journal of Economics. forthcoming

Bergstrom TC (2014) On the evolution of hoarding, risk-taking, and wealth distribution in nonhuman and human populations. Proc Natl Acad Sci 111(3):10860-10867

Bliss C, Nalebuff B (1984) Dragon slaying and ballroom dancing: the private supply of a public good. J Public Econ 25(1-2):1-12

Blomberg SB, Hess GD, Raviv Y (2009) Where have all the heroes gone? A rational-choice perspective on heroism. Public Choice 141(3-4):509-522

Böttcher MA, Nagler J (2016) Promotion of cooperation by selective group extinction. J Phys 18(6):063008

Diekmann A (1985) Volunteer's dilemma. J Confl Resolut 29(4):605-610

Eaton BC, Eswaran M (2003) The evolution of preferences and competition: a rationalization of Veblen's theory of invidious comparisons. Can J Econ 36(4):832-859

Eaton BC, Eswaran M, Oxoby RJ (2011) 'Us' and 'them': the origin of identity, and its economic implications. Canad J Econ 44(3):719-748

Eswaran M, Neary HM (2014) An economic theory of the evolutionary emergence of property rights. Am Econ J - Microecon 6(3):203-226

Foster D, Young P (1990) Stochastic evolutionary game dynamics. Theor Popul Biol 38(2):219-232

Freidlin MI, Wentzell AD (1984) Random perturbations of dynamical systems. Springer, New York

Fudenberg D, Imhof LA (2006) Imitation processes with small mutations. J Econ Theory 131(1):251-262

Fudenberg D, Nowak MA, Taylor C, Imhof LA (2006) Evolutionary game dynamics in finite populations with strong selection and weak mutation. Theor Popul Biol 70(3):352-363

Fudenberg D, Tirole J (1986) A theory of exit in duopoly. Econometrica 54(4):943-960

Gansberg M (1964) Thirty-eight who saw murder didn't call the police, The New York Times, March 27, 1964, https:/www.nytimes.com/1964/03/27/archives/37-who-saw-murder-didnt-call-the-policeapathy-at-stabbing-of.html, accessed December 04, 2020 
Harper M, Fryer D (2016) Stationary stability for evolutionary dynamics in finite populations. Entropy 18(9):316, 1-25

Hauert C, Imhof LA (2012) Evolutionary games in deme structured, finite populations. J Theor Biol 299:106-112

Hehenkamp B, Possajennikov A, Guse T (2010) On the equivalence of Nash and evolutionary equilibrium in finite populations. J Econ Behav Org 73(2):254-258

Johnson JP (2002) Open source software: private provision of a public good. J Econ Manag Strategy 11(4):637-662

Konrad KA, Morath F (2012) Evolutionarily stable in-group favoritism and out-group spite in intergroup conflict. J Theor Biol 306:61-67

Konrad KA, Morath F (2016) Evolutionary determinants of war. Defence Peace Econ 27(4):520-534

Manning R, Levine M, Collins A (2007) The Kitty Genovese murder and the social psychology of helping: the parable of the 38 witnesses. Am Psychol 62(6):555-562

Mielke A, Crockford C, Wittig RM (2019) Snake alarm calls as a public good in sooty mangabeys. Anim Behav 158:201-209

Mohseni A (2019) Stochastic stability and disagreement between dynamics. Philos Sci 86(3):497-521

Moran PAP (1962) The statistical process of evolutionary theory. Clarendon Press, Oxford

Nowak MA (2006) Evolutonary dynamics: exploring the equations of life. The Belknap Press of Harvard University Press, Cambridge

Nowak MA, Sasaki A, Taylor C, Fudenberg D (2004) Emergence of cooperation and evolutionary stability in finite populations. Nature 428(6983):646-650

Olivola CY, Kim Y, Merzel A, Kareev Y, Avrahami J, Ritov I (2020) Cooperation and coordination across cultures and contexts: individual, sociocultural, and contextual factors jointly influence decision making in the volunteer's dilemma game. J Behav Decis Mak 33(1):93-118

Przepiorka W, Diekmann A (2018) Heterogeneous groups overcome the diffusion of responsibility problem in social norm enforcement. Plos One 13(11):e0208129

Schaffer ME (1988) Evolutionary stable strategies for a finite population and a variable contest size. J Theor Biol 132(4):469-478

Taylor C, Fudenberg D, Sasaki A, Nowak MA (2004) Evolutionary game dynamics in finite populations. Bull Math Econ 66(6):1621-1644

Traulsen A, Nowak MA (2006) Evolution of cooperation by multilevel selection. Proc Natl Acad Sci 103(29):10952-10955

Publisher's note Springer Nature remains neutral with regard to jurisdictional claims in published maps and institutional affiliations. 